\title{
LETTER TO THE EDITOR Clonal evolution is a prognostic factor for the clinical progression of monoclonal B-cell lymphocytosis
}

Blood Cancer Journal (2017) 7, e597; doi:10.1038/bcj.2017.77; published online 25 August 2017

Monoclonal B-cell lymphocytosis (MBL) has attracted intensive research as the prelude of chronic lymphoproliferative disorders, mainly chronic lymphocytic leukemia (CLL). ${ }^{1}$ According to current criteria, MBL is a preclinical condition characterized by monoclonal B-cell expansions at small concentrations $\left(<5 \times 10^{9}\right.$ cells $/$ l) in the peripheral blood of otherwise healthy individuals. ${ }^{2,3}$ It is now obvious that $\mathrm{MBL}$ is a highly heterogeneous entity regarding the immunophenotypic characteristics and the B-cell clone burden. ${ }^{4-6}$ In immunophenotypic terms, MBL is distinguished into three main categories: (i) CLL-like (CD5 ${ }^{+} \mathrm{CD} 20^{\mathrm{dim}} \mathrm{CD} 23^{+}$slg $^{\text {low }}$ ) accounting for $70-75 \%$ of all cases; (ii) atypical $\left(\mathrm{CD}^{+} \mathrm{CD} 20^{\text {bright } /+}\right)$, mainly $\mathrm{CD}_{23} 3^{\mathrm{dim} /-}$; and (iii) $\mathrm{CD} 5^{\text {neg }} \mathrm{MBL}^{2,3}$ Based on the number of monoclonal B-cells, MBL is divided into low and high-count, each with a clearly different clinical course. ${ }^{5-7}$ Low-count MBL is a nonprogressive entity with a normal absolute B-cell count, whereas high-count or 'clinical' MBL (cMBL) is characterized by absolute lymphocytosis and progresses to CLL at a rate of $\sim 1-2 \%$ per year.8,9

The prevalence of CLL-like MBL is $10-100$-fold higher than that of CLL, indicating that most cases-even within the high-count category-do not evolve to overt disease. ${ }^{8-11}$ However, the biological features and molecular events which may contribute to the transition into the clinical state are far from being completely elucidated. Particularly, the available information on the clinical progression of non CLL-like MBL is scanty. ${ }^{12,13}$ To improve our understanding of these processes we performed a prospective study on a large series of $227 \mathrm{cMBL}$ cases of all three phenotypic categories testing for possible changes occurring during the natural course of MBL. These changes together with all available clinical parameters were further evaluated for their potential role in lymphomagenesis during a long-term follow-up (median 76 months).

All individuals included, attended the outpatient clinics of our hospital between January 2001 and January 2015 and characterized as MBL of any type according to current diagnostic criteria. They had no history or evidence of a hematological/solid neoplasia or autoimmunity and provided written informed consent to use laboratory data for research studies. The study followed the rules of the Interval Review Board and adhered to the declaration of Helsinki.

To avoid a mixed cohort with low-count $M B L$, we excluded cases with fewer than $0.5 \times 10^{9}$ clonal $B$ cells/L. All subjects underwent a regular hematologic follow-up every 6-12 months (median number of follow-up visits: 5; range 2-16). The MBL phenotype was characterized at diagnosis by flow-cytometry and the same cytometric assessment was repeated in cases with evidence of disease progression. A detailed cytogenetic evaluation was performed by fluorescent in situ hybridization at presentation and repeated at least once for all individuals still at the MBL stage, no sooner than 18 months after initial testing (median interval to repetition: 26 months; range 18-48). Disease progression was based on B-cell cut-off limit of $>5 \times 10^{9}$ cells/l (persisting for 3 months), and/or an increase in bone marrow infiltration rate
$>20 \%$, and/or the appearance of lymphadenopathy/organomegaly, detected either clinically or on ultrasound/computed tomography scans. Differences in time-to-event analysis were evaluated by log-rank statistics and multivariate analysis was performed by Cox proportional hazard regression. In case of continuous variables, the optimal cutoff points predicting the progression to overt disease were defined using the receiveroperator characteristic curves.

The flow-cytometry analysis showed a CLL-like phenotype in 130 subjects $(57.3 \%)$, an atypical one in $42(18.5 \%)$ and a CD5 ${ }^{\text {neg }}$ one in 55 (24.2\%). The three groups showed differences in some biological and hematological features such as the increased expression levels of ZAP70 in the non-CLL-like groups, and the significant lower presence of B-cells in the $C D 5^{\text {neg }}$ group both in absolute counts and qualitatively when measured as a percentage (\%) of the total lymphocytes (Table 1). Our broad fluorescent in situ hybridization analysis revealed abnormalities in 124/227 CMBL cases (54.6\%) at the initial examination with del(13q14) being the most frequent $(72 / 227,31.7 \%)$. Overall, $C D 5^{\text {neg }} M B L$ exhibited less often cytogenetic aberrations than the other groups (36.4\% in $C D 5^{\text {neg }}$ vs $63.1 \%$ in CLL-like and $52.4 \%$ in atypical MBL, $P=0.038)$ and most importantly, each category showed a clearly distinct cytogenetic pattern. Particularly, biallelic del(13q14) as a sole abnormality and concurrent monoallelic/biallelic del(13q14) were found only in CLL-like cases, $t(11 ; 14)$ detected only in atypical and del(7q31) only in $\mathrm{CD}^{\text {neg }}$ clones. In contrast, trisomy 12 was the only common finding among the 3 groups, whereas no del(6q23) or $\mathrm{t}(18 \mathrm{q} 21)$ were detected in any of the analyzed samples.

In general, the usual cytogenetic aberrations detected in CLL or other chronic lymphoproliferations are regarded as independent genetic events, so the presence of multiple aberrations may be considered as signs of clonal evolution. The same holds also true for homozygous $13 \mathrm{q}$ deletions. ${ }^{14}$ Accordingly, $19 \mathrm{cMBL}$ cases, mostly of the CLL-like group, showed evidence of clonal evolution (13 with homozygous or concomitant mono/biallelic del(13q14) and six cases with multiple abnormalities). However, the genomic instability was further highlighted by the cytogenetic reevaluation performed in each participant, while still at the $\mathrm{CMBL}$ phase. Particularly, the cytogenetic re-evaluation revealed 14 cases (11 CLL-like, two atypical and one $C D 5^{\text {neg }}$ ) showing novel abnormalities which were absent at presentation. Most of these cases acquired del(13q14) or +12 on a previous normal background (six and three cases respectively); in one case the hemizygous del(13q14) evolved to concomitant hemizygous/ homozygous loss, in one cMBL the initial concomitant mono/ biallelic del(13q14) evolved to homozygous loss and in another case +12 appeared in addition to del(13q14) already detected at diagnosis. The thirteenth case included the novel appearance of del(13q14) in the pre-existing +12 and the last case acquired a gain in 18q21 locus in addition to del(13q14) found initially.

The prospective nature of this study and our long-term monitoring allowed us to evaluate parameters which have an important role in the clinical progression of MBL. To date, $78 \mathrm{cMBL}$ cases (34.3\%) have evolved to overt disease (46/130 CLL-like, $15 / 42$ atypical and $17 / 55 \mathrm{CD}^{\text {neg }}$ cases) and 27 of them (11.9\%) have required treatment. Of the three groups $C D 5^{\text {neg }} \mathrm{CMBL}$ had 
Table 1. Clinical, biological and hematological features of individuals enrolled

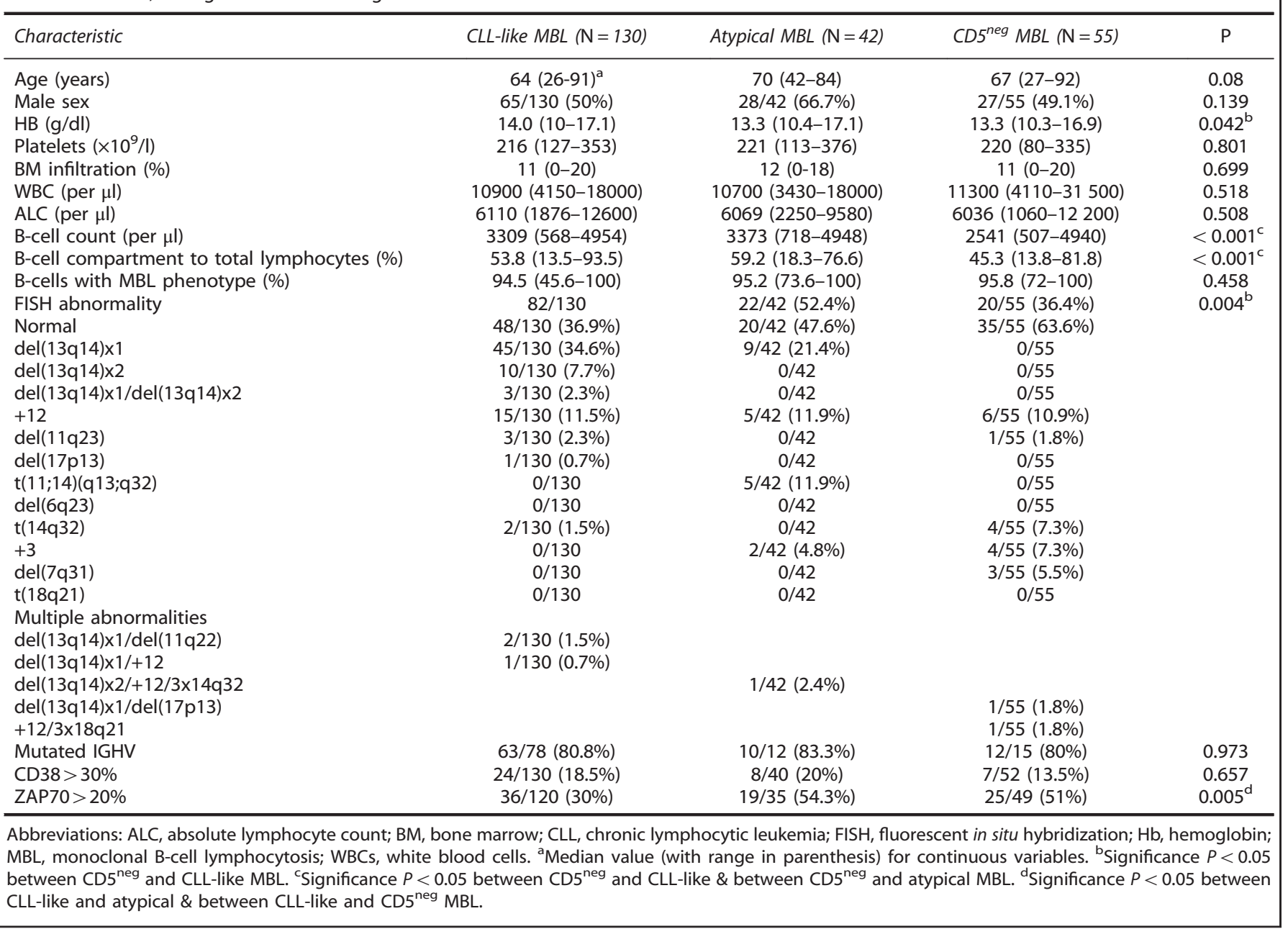

the most favorable profile (median time-to-progression: 84 months vs 66 months in CLL-like and 45 months in atypical, Figure 1a), which could be explained by the lower prevalence of genetic lesions compared to the other MBL subsets. Furthermore, the increased T/NK component in the $\mathrm{CD} 5^{\text {neg }}$ cases indicate an active role of microenvironmental 'bystander' immune cells that dynamically interact with the $\mathrm{CD} 5^{\text {neg }}$ cells and may restrain the clone in an indolent state. ${ }^{15}$

With regard to subgroup analysis, we searched for correlations between time-to-progression and clinical or biological features at diagnosis within each phenotypic subset. The variables tested were age, sex, WBC, ALC, B-cell count, T-cell count, platelet count, hemoglobin level, bone marrow infiltration rate, CD38 $\geqslant 30 \%$, ZAP70 $\geqslant 20 \%$, initial fluorescent in situ hybridization findings, immunoglobulin heavy chain variable region gene mutational status and the presence of clonal evolution. The higher B-cell count correlated with shorter time to disease progression in all phenotypic subsets, implying that common risk factors may operate in all different forms of the preclinical cMBL status. With the B-cell count treated as a continuous variable, we tried to define the optimal cutoff risk points in each category. A running log-rank test with a step-increase of 100 cells/ $\mu \mathrm{L}$ revealed two peaks in CLL-like MBL; cases with an initial B-cell count of more than 3900/ $\mu$ l carried a high risk of progression (median time: 37 months), those with a count of 2000-3900/ $\mu$ l had an intermediate risk (median time: 58 months), whereas those with a count below $2000 / \mu$ l showed a significantly lower probability (median: 116 months) $(P<0.001$ for all comparisons). Following the same approach, we found one peak of $3600 / \mu l$ for atypical $(P=0.007)$ and one peak of $3200 / \mu l$ for $C D 5^{\text {neg }} M B L$ cases $(P=0.005)$ (Figures $1 \mathrm{~b}-\mathrm{d})$.

Of the remaining variables, only clonal evolution was independently associated with a high risk of progression to clinical disease (hazards ratio: $2.25,95 \% \mathrm{Cl}: 1.16-4.38 \quad P=0.017$ ). Since clonal evolution was mainly detected in the CLL-like cases, risk analysis was restricted to this subset for better homogeneity, although statistical significance was not affected when all cases were included. In particular, individuals with clonal evolution had a shorter time-toprogression (median: 44 months), when compared to those who were cytogenetically stable (median: 82 months, $P<0.001$, Figure 1e). Interestingly, the presence of clonal evolution could clearly discriminate between two subgroups with significantly different risks of progression among patients at the intermediaterisk category according to B-cell count (2000-3900 B-cells/ $\mu \mathrm{l}$ ) (median time: 47 vs 77 months, $P<0.001$ ) (Figure 1f). Finally, the negative impact of this parameter was also identified in time-totreatment analysis, since cases with clonal evolution at the preclinical stage, showed both an increased rate for treatment requirement in our monitoring period and a significant shorter time-to-treatment when compared with the rest of the cohort (Figure 1g).

In summary, the three phenotypically defined MBL groups have distinct characteristics, but seem to share common features involved in clinical progression. Certain chromosome aberrations occur early in the transformation process but do not seem to affect the risk of progression to the clinical state. On the other hand, the apparent genomic instability, manifested by the 

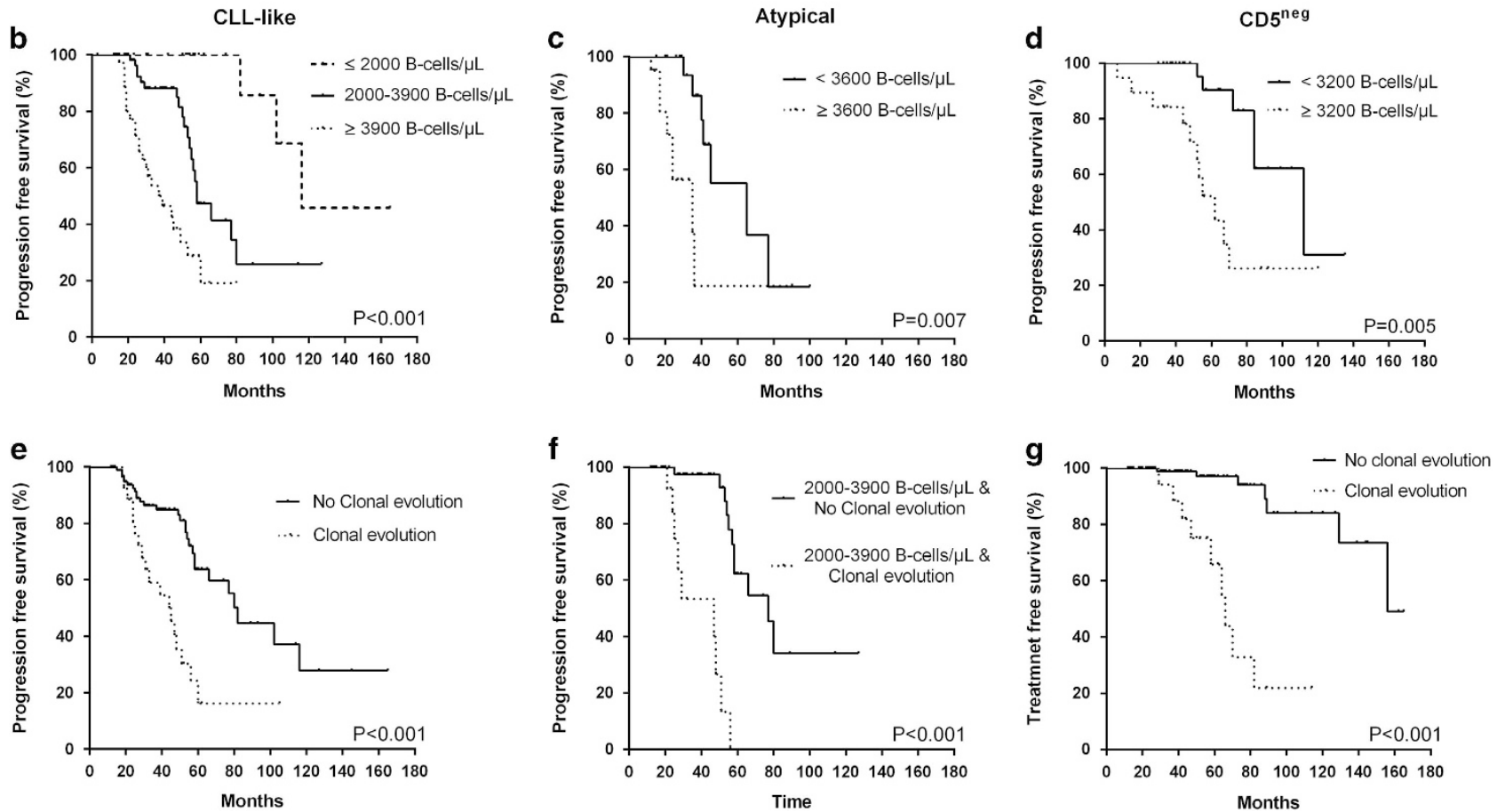

Figure 1. Risk of progression from MBL to CLL or other CLPDs. (a) Time-to-progression according to the phenotypic category. (b-d) Progression-free survival according to absolute B-cell count for CLL-like (b), atypical (c) and CD5 ${ }^{\text {neg }}$ (d) MBL. (e) Progression-free survival according to the presence of clonal evolution. (f) Progression-free survival for CLL-like MBL subjects with 2000-3900 B-cell count at initial diagnosis according to the presence or absence of clonal evolution. (g) Treatment-free survival for progressed cases according to the presence of clonal evolution at the preclinical MBL stage. CLPD, chronic lymphoproliferative disorder.

acquisition of additional abnormalities, together with increased initial B-cell counts are the strongest determinants of disease progression. The combination of these two variables could better stratify MBL subjects into more precise prognostic subgroups.

\section{CONFLICT OF INTEREST}

The authors declare no conflict of interest.

\section{ACKNOWLEDGEMENTS}

We thank all clinicians who enrolled $\mathrm{MBL}$ individuals in this prospective study. We also thank Kostas Papadimitriou for his help in sorting experiments.

IV Kostopoulos ${ }^{1,2}$, G Paterakis ${ }^{3}$, D Pavlidis ${ }^{1}$, E Kastritis $^{4}$, E Terpos ${ }^{4}$, OE Tsitsilonis ${ }^{2}$ and SI Papadhimitriou'

${ }^{1}$ Hematology Laboratory, 'G. Gennimatas' Athens General Hospital, Athens, Greece;

${ }^{2}$ Section of Animal and Human Physiology, Department of Biology, National and Kapodistrian University of Athens, Athens, Greece;

${ }^{3}$ Flow Cytometry Laboratory, 'G. Gennimatas' Athens General Hospital, Athens, Greece and
${ }^{4}$ Department of Clinical Therapeutics, School of Medicine, National and Kapodistrian University of Athens, Athens, Greece

E-mail: gikosto@gmail.com

\section{REFERENCES}

1 Landgren O, Albitar M, Ma W, Abbasi F, Hayes RB, Ghia P et al. B-cell clones as early markers for chronic lymphocytic leukemia. N Engl J Med 2009; 360: 659-667.

2 Marti GE, Rawstron AC, Ghia P, Hillmen P, Houlston RS, Kay N et al. Diagnostic criteria for monoclonal B-cell lymphocytosis. Br J Haematol 2005; 130: 325-332.

3 Hallek M, Cheson BD, Catovsky D, Caligaris-Cappio F, Dighiero G, Dohner $\mathrm{H}$ et al. Guidelines for the diagnosis and treatment of chronic lymphocytic leukemia: a report from the International Workshop on Chronic Lymphocytic Leukemia updating the National Cancer Institute-Working Group 1996 guidelines. Blood 2008; 111: 5446-5456.

4 Shim YK, Middleton DC, Caporaso NE, Rachel JM, Landgren O, Abbasi F et al. Prevalence of monoclonal B-cell lymphocytosis: a systematic review. Cytometry $B$ Clin Cytom 2010; 78: S10-S18.

5 Rawstron AC, Shanafelt T, Lanasa MC, Landgren O, Hanson C, Orfao A et al. Different biology and clinical outcome according to the absolute numbers of clonal B-cells in monoclonal B-cell lymphocytosis (MBL). Cytometry B Clin Cytom 2010; 78: S19-S23. 
6 Kostopoulos IV, Paterakis G, Papadimitriou K, Pavlidis D, Tsitsilonis OE, Papadhimitriou SI. Immunophenotypic analysis reveals heterogeneity and common biologic aspects in monoclonal B-cell lymphocytosis. Genes Chromosomes Cancer 2015; 54: 210-221.

7 Fazi C, Scarfo L, Pecciarini L, Cottini F, Dagklis A, Janus A et al. General population low-count CLL-like MBL persists over time without clinical progression, although carrying the same cytogenetic abnormalities of CLL. Blood 2011; 118: 6618-6625.

8 Rawstron AC, Bennett FL, O'Connor SJ, Kwok M, Fenton JA, Plummer M et al. Monoclonal B-cell lymphocytosis and chronic lymphocytic leukemia. N Engl J Med 2008; 359: 575-583.

9 Shanafelt TD, Kay NE, Rabe KG, Call TG, Zent CS, Maddocks K et al. Brief report: natural history of individuals with clinically recognized monoclonal B-cell lymphocytosis compared with patients with Rai 0 chronic lymphocytic leukemia. J Clin Oncol 2009; 27: 3959-3963.

10 Nieto WG, Almeida J, Romero A, Teodosio C, Lopez A, Henriquez AF et al. Increased frequency (12\%) of circulating chronic lymphocytic leukemia-like B-cell clones in healthy subjects using a highly sensitive multicolor flow cytometry approach. Blood 2009; 114: 33-37.

11 Rossi D, Sozzi E, Puma A, De Paoli L, Rasi S, Spina V et al. The prognosis of clinical monoclonal B cell lymphocytosis differs from prognosis of Rai 0 chronic lymphocytic leukaemia and is recapitulated by biological risk factors. $\mathrm{Br} \mathrm{J}$ Haematol 2009; 146: 64-75.

12 Nieto WG, Teodosio C, Lopez A, Rodriguez-Caballero A, Romero A, Barcena P et al. Non-CLL-like monoclonal B-cell lymphocytosis in the general population: prevalence and phenotypic/genetic characteristics. Cytometry B Clin Cytom 2010; 78: S24-S34.

13 Amato D, Oscier DG, Davis Z, Mould S, Zheng J, Kolomietz E et al. Cytogenetic aberrations and immunoglobulin $\mathrm{VH}$ gene mutations in clinically benign CD5- monoclonal B-cell lymphocytosis. Am J Clin Pathol 2007; 128: 333-338.

14 Chena C, Avalos JS, Bezares RF, Arrossagaray G, Turdó K, Bistmans A et al. Biallelic deletion $13 q 14.3$ in patients with chronic lymphocytic leukemia: cytogenetic, FISH and clinical studies. Eur J Haematol 2008; 81: 94-99.

15 Rosenquist R, Davi F, Ghia P. The microenvironment in lymphomas--dissecting the complex crosstalk between tumor cells and 'by-stander' cells. Semin Cancer Biol 2014; 24: 1-2.

\section{cc) (i)}

This work is licensed under a Creative Commons Attribution 4.0 International License. The images or other third party material in this article are included in the article's Creative Commons license, unless indicated otherwise in the credit line; if the material is not included under the Creative Commons license, users will need to obtain permission from the license holder to reproduce the material. To view a copy of this license, visit http://creativecommons.org/licenses/ by/4.0/

(c) The Author(s) 2017 